\title{
Erratum to: Transfersomal Nanoparticles for Enhanced Transdermal Delivery of Clindamycin
}

\author{
Ahmed A. H. Abdellatif ${ }^{1,3}$ and Hesham M. Tawfeek ${ }^{2}$
}

\section{Erratum to: AAPS PharmSciTech \\ DOI 10.1208/s12249-015-0441-7}

While the captions of Figs. 5 and 6 are correct in the published article, the content of Figs. 5 and 6 were inadvertently switched during production. The correct figures with the correct captions appear below.

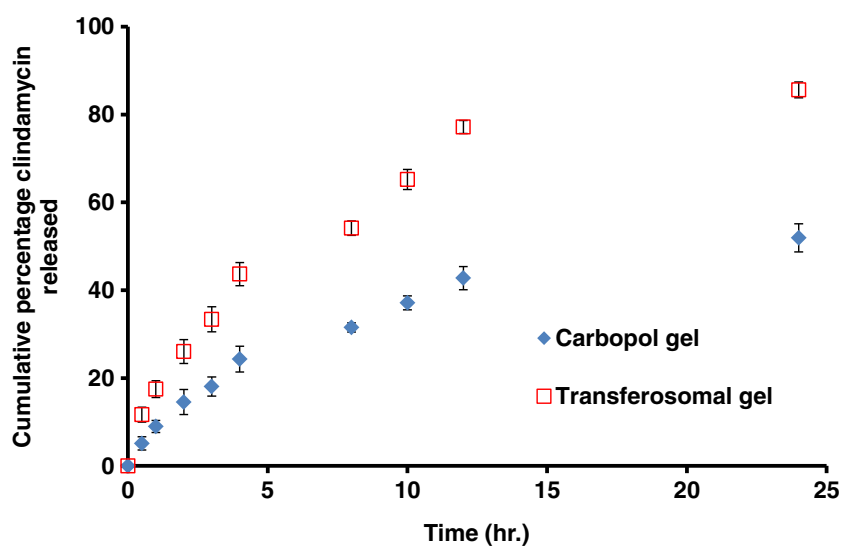

Fig. 5. In vitro drug release studies of transfersomal gel formulations using a cellophane membrane. In vitro clindamycin release has been performed in apparatus resemble to Franz diffusion cell. The tube was immersed vertically in a $100 \mathrm{~mL}$ beaker containing $50 \mathrm{~mL}$ of phosphate buffer $(\mathrm{pH}$ 7.4). The transfersomal gel formulation $(1 \mathrm{~g}$ equivalent to $50 \mathrm{mg}$ ) was placed into the glass tube. At predetermined time intervals for up to $24 \mathrm{hrs}$, $5 \mathrm{ml}$ aliquots of the release medium were withdrawn for analysis and were replaced with equal volume of release medium, phosphate buffer, at the same temperature to maintain constant volume.

The online version of the original article can be found at http:/ dx.doi.org/10.1208/s12249-015-0441-7.

\footnotetext{
${ }^{1}$ Department of Pharmaceutics and Industrial Pharmacy, Faculty of Pharmacy, Al-Azhar University, Assuit, Egypt.

${ }^{2}$ Department of Industrial Pharmacy, Faculty of Pharmacy, Assiut University, Assuit, Egypt.

${ }^{3}$ To whom correspondence should be addressed. (e-mail: ahmed.a.h.abdellatif@azhar.edu.eg)
}

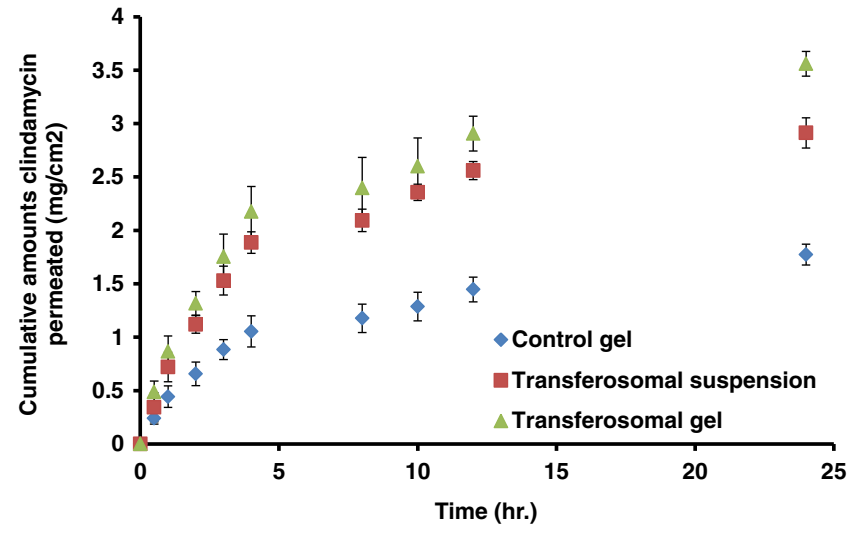

Fig. 6. Ex vivo of the formulated transfersomal gel through rat skin and compared with both control gel and transfersomal suspension. The permeation study of clindamycin from transfersomal gel formulations was carried out using abdominal male rat skin $(150 \pm 25 \mathrm{~g})$.

There is also an error in the "Methods" section of the published article. In the next to the last sentence of the first paragraph ("Formulation of Plain and Clindamycin-Loaded TRSs") "aqueous" should be "organic". 